\title{
A Survey of Current Treatment Practices for Postpartum Hemorrhage by Practicing Obstetricians and Hematologists
}

\author{
Elizabeth W. Triche ${ }^{1 * \#, ~ M a r k ~ J . ~ W e h r u m ~}{ }^{2 *}$, Michael J. Paidas ${ }^{3}$ \\ ${ }^{1}$ Department of Epidemiology, Brown University School of Public Health, Providence, USA \\ ${ }^{2}$ Department of Obstetrics \& Gynecology, Womack Army Medical Center, Fort Bragg, NC, USA \\ ${ }^{3}$ Yale Women and Children's Center for Blood Disorders, Department of Obstetrics, Gynecology and \\ Reproductive Sciences, Division of Maternal Fetal Medicine, Yale University, New Haven, USA \\ Email: "Elizabeth triche@brown.edu, mark.j.wehrum.mil@mail.mil, Michael.paidas@yale.edu
}

Received 3 February 2014; revised 1 March 2014; accepted 8 March 2014

Copyright (C) 2014 by authors and Scientific Research Publishing Inc.

This work is licensed under the Creative Commons Attribution International License (CC BY). http://creativecommons.org/licenses/by/4.0/

(c) (i) Open Access

\section{Abstract}

Objective: To identify current treatment strategies for postpartum hemorrhage used by obstetricians (OB/GYNs) and hematologists (HEMs). Study Design: We conducted a survey of OB/GYNs ( $n=$ 220) and HEMs $(n=30)$ to describe the characteristics of current treatment strategies for postpartum hemorrhage. Surveys were administered via a structured questionnaire on a secure internet website from 5 - 12 October 2009. Results: The majority of OB/GYN and HEM respondents were practicing in a community hospital environment (77\%). Of the OB/GYNs, the majority practiced at hospitals with over 2000 deliveries per year (77\%). A majority (58\%) of OB/GYNs were affiliated with hospitals that lacked a massive transfusion protocol to treat severe postpartum hemorrhage. Subsequent to uterine massage and additional oxytocin, the majority of $0 B / G Y N s$ (73\%), preferred the administration of Methergine ${ }^{\circledR}$ as the next level of intervention for postpartum hemorrhage. There was considerable variability in response to specific treatment strategies for several hypothetical case scenarios; however, the large majority of $0 B / G Y N s$ favored obstetrical procedures over interventional radiology or administration of rFVIIa. A large majority (77\%) of physicians who are familiar with rRVIIa as treatment for postpartum hemorrhage reported being very satisfied with the agent for this indication. Conclusions: An established, systematic treatment strategy among $\mathrm{OB} / \mathrm{GYNs}$ emerged only in the case of mild postpartum hemorrhage.

\footnotetext{
*Authorship equally shared.

\#Corresponding author.
} 


\section{Keywords}

\section{Postpartum Hemorrhage; Physician Survey; Recombinant Factor VIIa; Clinical Management}

\section{Introduction}

Acute postpartum hemorrhage is the leading cause of global maternal mortality, and a principal contributor to maternal morbidity [1]. The World Health Organization estimates that 530,000 women die each year from pregnancy-related causes [2]; approximately $25 \%$ of these maternal deaths are due to severe bleeding [3]. In the spectrum of obstetrical emergencies, massive obstetrical hemorrhage and shoulder dystocia are two of the most anxiety provoking situations encountered by obstetrical care providers. While recommendations for the management of shoulder dystocia have not changed greatly in recent years, the effective management of massive obstetrical hemorrhage has evolved to include a variety of medical and surgical strategies as well as standardized institutional approaches shown to improve outcomes [4]. Whereas the management of shoulder dystocia can be successfully managed by an algorithmic approach, massive obstetrical hemorrhage requires individualized management of each patient to decrease maternal morbidity and mortality.

Proper preparation and resources to manage maternal hemorrhage in a timely manner can be lifesaving [5]. Typical medical management of postpartum hemorrhage may include use of uterotonic agents; non-surgical maneuvers such as uterine packing, balloon catheter, or embolization procedures [6]; fertility-preserving surgical procedures such as artery ligation, or B-Lynch suture; or if these procedures fail, puerperal hysterectomy. The ability of an obstetrical provider to effectively manage patients with postpartum hemorrhage is largely dependent on the individual's residency training, personal experience and familiarity with the recommendations and controversies in the most current literature. For example, there is longstanding debate on whether crystalloid or colloid solutions are the best in the initial fluid resuscitation [7]-[9]. In addition, there is no consensus amongst authorities as to what represents the appropriate hemoglobin threshold for transfusion [10] or how rapidly and in what form to transfuse blood products [11] [12]. Recently, the use of rFVIIa has been reported in the management of life-threatening postpartum hemorrhage [13]-[19], although it is only currently FDA-approved for episodes of severe hemorrhage or perioperative management of bleeding in patients with congenital factor VII deficiency and hemophilia A or B with inhibitors [20]. As a result, there is a wide variety of management practices for postpartum hemorrhage amongst obstetrical providers in the United States. Massive transfusion protocols exist in some institutions with accredited trauma centers [21]. However, the most common scenario involves the obstetric provider, managing a case of postpartum hemorrhage based his or her own practice habits on perhaps the assistance of Maternal Fetal Medicine or Hematology consultant.

There is a dearth of literature investigating the management of postpartum hemorrhage, particularly addressing national trends. Given that postpartum hemorrhage is an increasing problem in obstetrical practice and that there are very few studies attempting to clarify its optimal management, we conducted a survey of OB/GYNs and a smaller number of HEMs to identify current treatment strategies for postpartum hemorrhage. In addition to obstetrical strategies, we were interested in the current use of non-obstetrical strategies including rFVIIa for postpartum hemorrhage, and how these practices varied between OB/GYNs and HEMs, and by practice characteristics (e.g., obstetrical patient volume, medical specialty, years in practice, etc.) and basic demographics (e.g., age and gender).

\section{Materials and Methods}

The study was conducted after the Yale University Institutional Review Board (IRB) granted an exemption to the IRB review process. A comprehensive electronic database review was performed, using PUBMED and MEDLINE to search for studies published from 1975 to 2010, investigating national trends of postpartum hemorrhage management. Examples of MeSH terms used for this literature search included postpartum hemorrhage, pregnancy and health care surveys. A total of nine references that addressed the issue of postpartum hemorrhage management in a survey format were identified. None of the identified references addressed the national trends of postpartum management in the United States and only one of the references dealt with current evidence-based practice and recommendations [22]. Therefore, we conducted a survey developed by one of the 
authors (MJP) of a national sample of obstetricians (OB/GYNs) to describe the current treatment strategies relative to postpartum hemorrhage. While the primary focus was on OB/GYNs, we also included a smaller sample of hematologists (HEMs) because they are often consulted when bleeding issues arise. Funding for this survey was provided by Novo Nordisk ${ }^{\circledR}$ (Bagsvaerd, Denmark).

A sample of OB/GYNs and HEMs was randomly drawn from a national database of these physicians maintained by the American Medical Association (AMA). Participants were offered an honorarium to encourage their participation. The surveys were administered by Psymed Inc. (Valley Forge, PA), via a semi-structured questionnaire on a secure internet website from 5 - 12 October 2009. (See Appendix 1 for survey questions). In order to be eligible to participate in the survey, physicians had to indicate that they were in full-time practice, have board certification in their primary specialty and be involved in or asked to consult on at least one case of postpartum hemorrhage associated with a vaginal or cesarean delivery within the past two years. For OB/GYNs to participate in this survey, they also had to have a practice which included at least $20 \%$ obstetrical patients, personally deliver at least some of their own patients, and manage or consult on at least one case of postpartum hemorrhage per year.

A total of 1800 electronic physician surveys were distributed but an a priori target sample size of $200 \mathrm{OB} /$ GYNs was determined necessary to describe obstetrical practices in response to $\mathrm{PPH}$; after that target was reached, the survey site was closed to further participants. At that time, a total of 285 physicians had responded to the electronic survey. Thirty-five of the physician surveys were incomplete or did not satisfy eligibility criteria, resulting in a final sample in the analysis of 250 physicians, of whom 220 were OB/GYNs and 30 HEMs.

The survey gathered information on practice characteristics (e.g., obstetrical patient volume, medical specialty, years in practice, etc.) and basic demographics (e.g., age, gender) of each respondent while also eliciting specific postpartum hemorrhage management strategies employed. The questions pertaining to the management of postpartum hemorrhage comprehensively addressed the areas of medical and surgical management as well as the strategy for administration of blood products and the use of rFVIIa.

The survey was pre-tested by two physicians who have expertise in obstetrics and postpartum hemorrhage. The questionnaire was then modified based on their feedback. Questions pertaining to the demographics and the practice of the respondents were left open-ended to allow for a wide variety of descriptions. Open-ended responses were examined and then manually coded for key themes. Questions regarding the management of postpartum hemorrhage were closed-ended with options pertaining to specific decisions or interventions the respondent favored. The survey strategy was implemented to provide a more detailed representation of the respondents' practice strategies. Descriptive statistics were tabulated and measures of association were calculated using $\chi^{2}$ analysis for categorical variables and the Student $t$ test for continuous variables.

\section{Results}

As described in Table 1, this sample includes 220 OB/GYNs and 30 HEMs. There were 50 (20\%) respondents that had additional training certifications (Internal Medicine, Maternal Fetal Medicine and Oncology). The majority of respondents were practicing in a community hospital environment $(n=193,77 \%)$. Of the OB/GYN respondents, the majority practiced at hospitals that reported over 2000 deliveries per year $(n=170,77 \%)$. Nearly three-quarters $(74 \%)$ of the physicians were male. The mean age of respondents was 47.7 (s.d. $=7.5$ ), with a range of 30 - 66 years of age.

A majority (58\%) of OB/GYNs were affiliated with hospitals that lacked a massive transfusion protocol to treat severe postpartum hemorrhage; $42 \%$ responded that their hospitals did have an established protocol (Figure 1). Public university hospitals were reportedly more likely to have an established protocol (67\%) than private university (49\%) or community hospitals (35\%). As the number of annual deliveries increased at an institution, so did the propensity to have an established massive transfusion protocol (Figure 1).

A small number of OB/GYNs (10\%) report having no access to advanced interventions to treat postpartum hemorrhage available to them at their institutions (Table 2). Overall, $71 \%$ of OB/GYNs reported that they have the expertise to perform B-Lynch suture or a similar compression technique. In regard to nonsurgical options available to OB/GYNs, $65 \%$ had Interventional Radiology (IR) available to perform uterine artery embolization and $61 \%$ reported the ability to insert a Bakri balloon for uterine tamponade (Table 2). Only 27\% of OB/GYNs reported the ability to administer rFVIIa.

As part of the eligibility criteria, physicians were required to report being involved with a minimum of one 
Table 1. Demographic characteristics of respondents to a national survey of postpartum hemorrhage management.

\begin{tabular}{|c|c|c|c|c|}
\hline & Number in Sample (n) & Proportion of Sample (\%) & & \\
\hline Total & 250 & $100 \%$ & & \\
\hline \multicolumn{5}{|l|}{ Medical specialty } \\
\hline OB/GYNs & 220 & $88 \%$ & & \\
\hline HEMs & 30 & $12 \%$ & & \\
\hline \multicolumn{5}{|l|}{ Additional medical certifications } \\
\hline Additional Certifications & 50 & $20 \%$ & Internal Medicine & $12 \%$ \\
\hline \multirow[t]{2}{*}{ No Additional Certifications } & 200 & $80 \%$ & Maternal Fetal Medicine & $5 \%$ \\
\hline & & & Oncology & $3 \%$ \\
\hline \multicolumn{5}{|l|}{ Hospital affiliation } \\
\hline Private university medical center & 41 & $16 \%$ & & \\
\hline Public university medical center & 15 & $6 \%$ & & \\
\hline Community hospital & 192 & $77 \%$ & & \\
\hline \multirow[t]{2}{*}{ Other } & 2 & $1 \%$ & Academic medical center & $n=1$ \\
\hline & & & $\begin{array}{l}\text { Non-profit hospital/ } \\
\text { university associated }\end{array}$ & $n=1$ \\
\hline \multicolumn{5}{|l|}{ Obstetrical volume (OB/GYNs only) } \\
\hline$<2000$ deliveries/year & 50 & $23 \%$ & & \\
\hline 2000 - 5000 deliveries/year & 117 & $53 \%$ & & \\
\hline 5000 - 10,000 deliveries/year & 46 & $21 \%$ & & \\
\hline$>10,000$ deliveries/year & 7 & $3 \%$ & & \\
\hline \multicolumn{5}{|l|}{ Gender } \\
\hline Male physicians & 184 & $74 \%$ & & \\
\hline Female physicians & 66 & $26 \%$ & & \\
\hline Age $(\mu$, s.d. $)$ & $47.7 \pm 7.5$ & & & \\
\hline
\end{tabular}

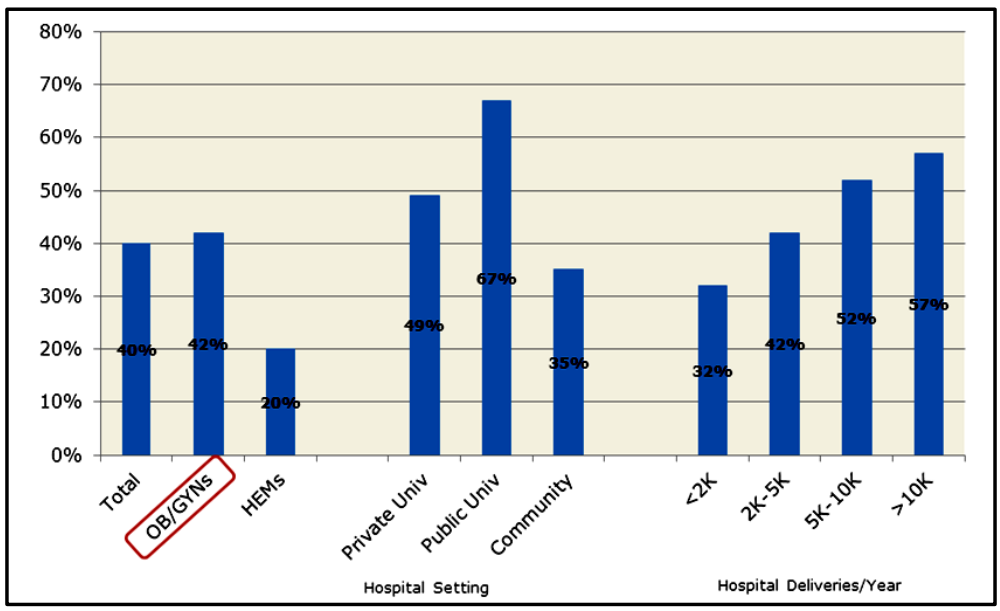

Figure 1. Physicians reporting an established massive transfusion protocol to treat severe hemorrhage (\% physicians). 
Table 2. Interventions readily available at OB/GYNs' institutions ( $n=220$ OB/GYNs).

\begin{tabular}{cc}
\hline Intervention* & OB/GYN providers, $\boldsymbol{n}$ (\%) \\
\hline Expertise to perform B-Lynch & $156(71 \%)$ \\
Interventional radiology available to perform uterine artery embolization or balloon inflation procedure & $143(65 \%)$ \\
Placement of Bakri balloon & $134(61 \%)$ \\
Administration or rVIIa & $59(27 \%)$ \\
No interventions available & $22(10 \%)$ \\
\hline
\end{tabular}

*Question not posed to HEM's.

case of postpartum hemorrhage annually. OB/GYNs meeting this criterion were involved with a median of 12 total cases of postpartum hemorrhage annually, while HEMs reported being involved with about 5.5 cases annually. Over the past three years, OB/GYNs reported a median of 6 cases and HEMs a median of 7 cases of severe PPH. There was a gradient in median number of total cases of PPH by hospital type with highest numbers in private university hospitals and lowest in community hospitals. For severe cases, private university hospitals had the highest number (median $=10$ ) while public university and community hospitals had similar numbers (median $=6$ ) (Figure 2). Mean values tended to be positively skewed.

Less than half (45\%) of patients that have a documented postpartum hemorrhage are currently reportedly receiving hematological evaluations; only $41 \%$ of OB/GYN PPH patients compared to $80 \%$ of HEM patients receive an evaluation. When evaluated, $20 \%$ of patients under care of OB/GYNs were found to have a bleeding disorder, compared to $58 \%$ of patients evaluated by a hematologist (Figure 3).

Nearly all OB/GYNs had seen PPH due to uterine atony in the vaginal (97\%) and cesarean delivery (96\%) settings (Table 3). In the vaginal delivery setting, the majority of OB/GYNs reported seeing PPH secondary to retained placenta (94\%), retained blood clots (75\%) and genital tract trauma (66\%), but fewer reported these in the setting of cesarean deliveries. Other settings in which OB/GYNs had encountered PPH were placenta accreta, increta or percreta; placenta previa; and pregnancy complications associated with coagulopathy, including preeclampsia disorders, fetal death.

HEMs most frequently provided consultation on OB PPH patients when coagulation factors were involved: complications related to coagulation disorders (67\%); pregnancy complications associated with coagulopathy (47\%); or complications related to anticoagulation use (43\%) (Figure 4). Despite being the most common situation in which OB/GYNs have encountered PPH, only 30\% of HEMs report being consulted about patients with PPH secondary to uterine atony.

\subsection{Current Treatment Practices}

As part of the survey, physicians were presented with several progressively severe PPH case scenarios pertaining to management strategies that ultimately required invasive interventional procedures and/or transfusion of blood products, and were asked to report their "likely approach to treatment." Some questions were posed with the assumption that interventional radiology for embolization and rFVIIa were both available in their hospital setting. As scenarios progressed in severity, management strategies began to diverge with no clear majority responses predominating, unlike for mild PPH. Current treatment strategies are summarized in Table 4.

In the setting of mild PPH (Estimated Blood Loss $(\mathrm{EBL})=1$ liter) secondary to uterine atony (Appendix 1, Q \#24), the vast majority of OB/GYNs (92\%) identify uterine massage as the primary treatment strategy along with continuing oxytocin and administering IV volume replacement. Subsequent to uterine massage and additional oxytocin, a large majority of OB/GYNs (73\%), preferred the administration of Methergine ${ }^{\circledR}$ as the next level of intervention for postpartum hemorrhage $(E B L=1$ liter) (Table 4). Other uterotonic agents reportedly used as "next steps" following uterine massage and oxytocin included the administration of misoprostol per rectum (13\%) and Hemabate ${ }^{\circledR}(11 \%)$.

In the event that uterotonic medications were unsuccessful and postpartum hemorrhage continued (EBL $=1.5$ liters) (Appendix 1, Q \#26), 62\% of OB/GYNs reported that their "next step” in intervention would be insertion of a Bakri balloon into the uterine cavity, followed by consultation with Interventional Radiology for arterial 


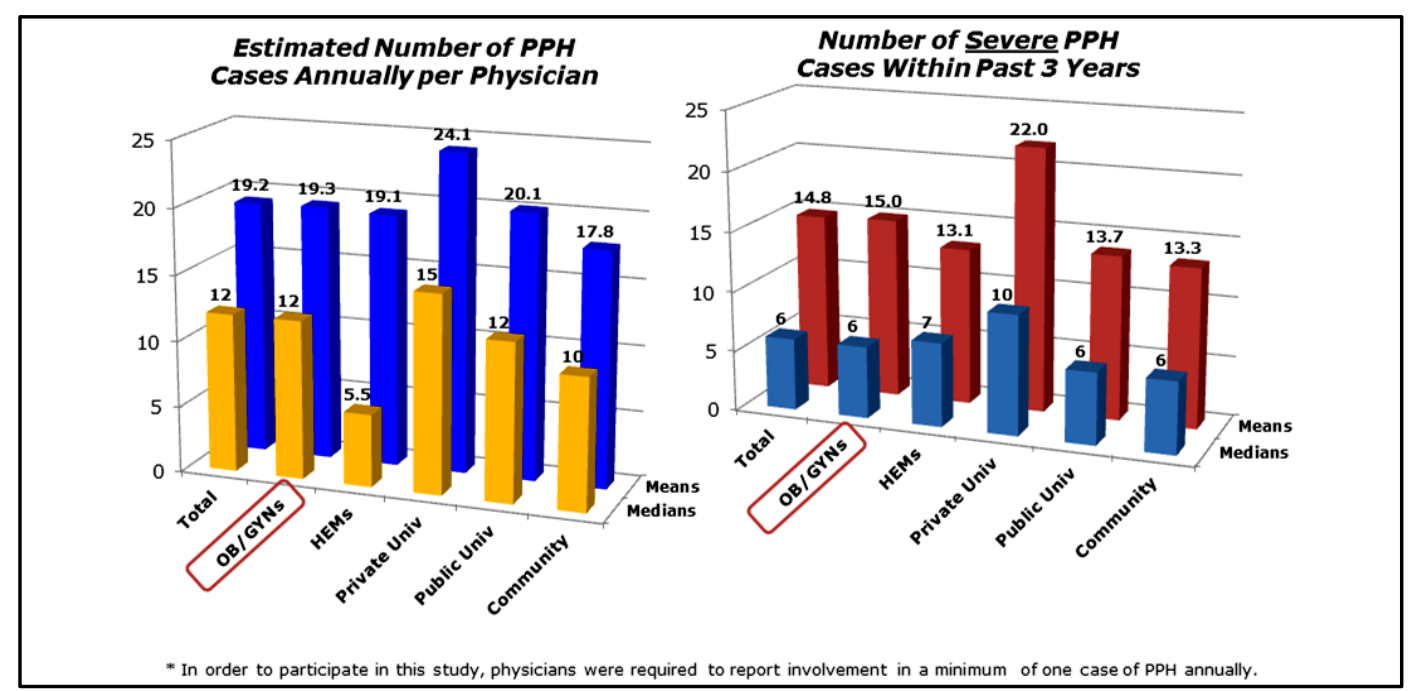

Figure 2. Estimated total number of annual PPH cases and number of severe PPH cases over past three years.

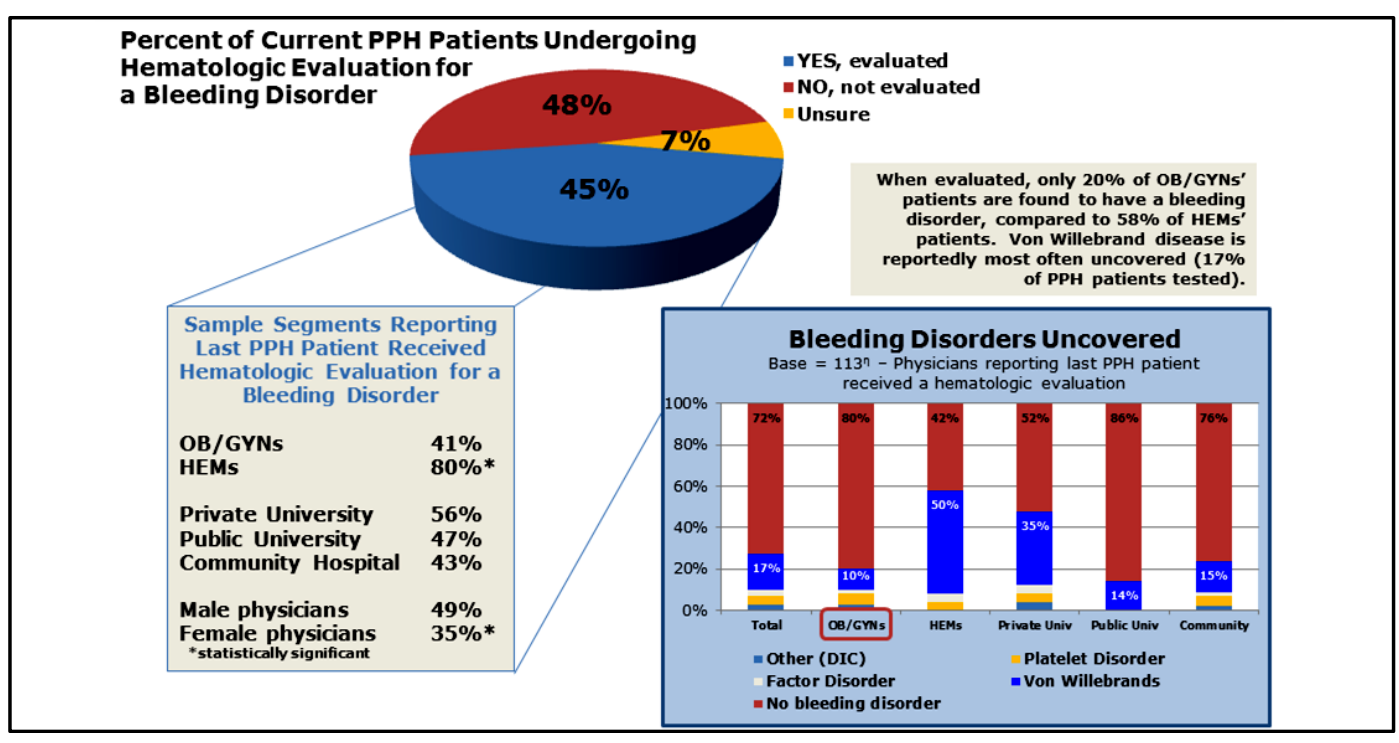

Figure 3. Hematologic evaluations for PPH patients.

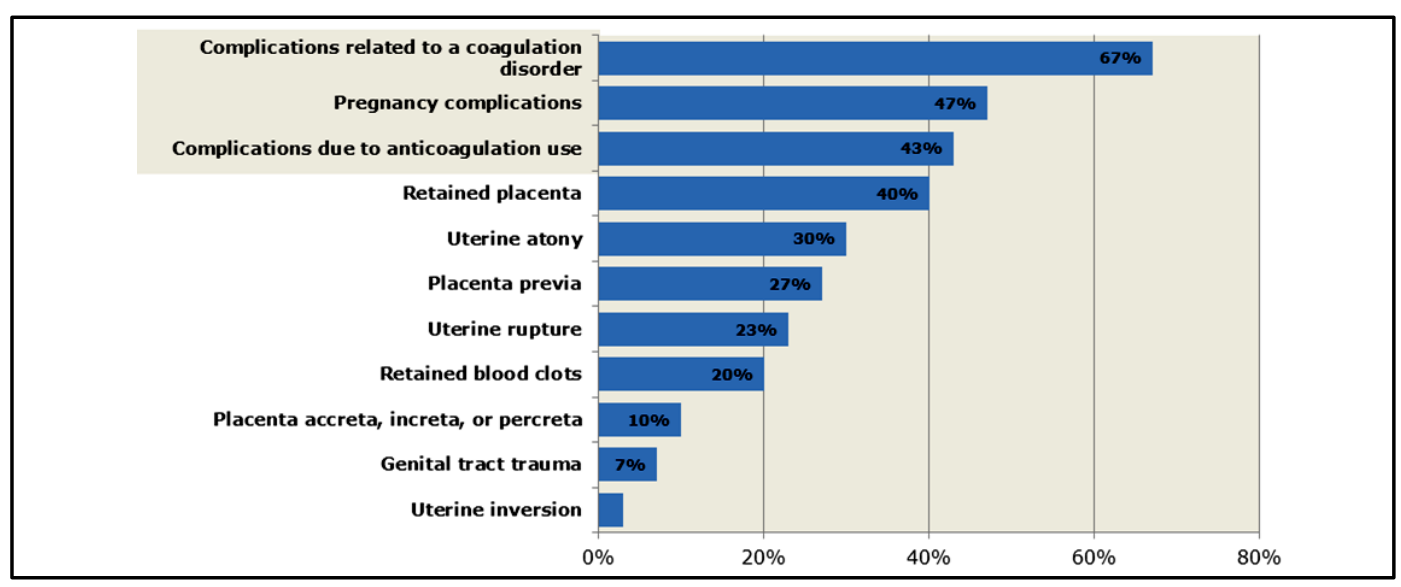

Figure 4. Settings in which HEMs have provided consultation on obstetrical PPH Patients ( $n=30$ HEMs only). 
Table 3. Settings in which OB/GYNs have encountered postpartum hemorrhage (\% of OB/GYNs reporting).

\begin{tabular}{ccc}
\hline Setting & Vaginal & Cesarean \\
Uterine atony & $\mathbf{9 7 \%}$ & $\mathbf{9 6 \%}$ \\
Retained placenta & $\mathbf{9 4 \%}$ & $29 \%$ \\
Retained blood clots & $\mathbf{7 5 \%}$ & $36 \%$ \\
Genital tract trauma & $\mathbf{6 6 \%}$ & $\mathbf{8 1 \%}$ \\
Placenta accreta, increta or percreta & $\mathbf{6 4 \%}$ & $\mathbf{7 3 \%}$ \\
Placenta previa & $38 \%$ & $\mathbf{6 0 \%}$ \\
\hline $\begin{array}{c}\text { Pregnancy complications associated with coagulopathy, } \\
\text { increasing preeclampsia disorders, fetal death }\end{array}$ & $\mathbf{5 8 \%}$ & \\
\hline
\end{tabular}

Table 4. Summary of hypothetical PPH case scenarios and reported OB/GYN treatment strategies.

\begin{tabular}{|c|c|c|c|}
\hline $\begin{array}{l}\text { Situation: } P P H \text { presentations progress in } \\
\text { severity and estimated blood loss }(E B L)\end{array}$ & OB/GYNs' Treatment Strategy & $\begin{array}{l}\text { Frequency/Variation } \\
\text { Among OB/GYNs }\end{array}$ & Question \# and Salient Facets \\
\hline \multirow[t]{2}{*}{$\begin{array}{l}\text { Vaginal delivery; PPH secondary to } \\
\text { uterine atony; EBL = } 1 \text { liter }\end{array}$} & $\begin{array}{l}\text { Uterine massage; continue oxytocin; } \\
\text { give IV volume replacement }\end{array}$ & Vast majority (96\%) & Q \#23 \\
\hline & $\begin{array}{l}\text { NEXT STEP: Administer } \\
\text { Methergine }^{\circledR} 0.2 \mathrm{mg} \text { intramuscularly }\end{array}$ & Large majority (73\%) & $\mathrm{Q} \# 24$ \\
\hline $\begin{array}{l}\text { Vaginal delivery; PPH secondary to } \\
\text { uterine atony; all uterotonics administered; } \\
\text { PPH persists; EBL=1.5 liters }\end{array}$ & $\begin{array}{l}\text { Bakri balloon placement } \\
\text { Other interventions }\end{array}$ & $\begin{array}{l}\text { Majority (62\%) } \\
\text { 38\% }\end{array}$ & $\begin{array}{l}\text { Q \#26 } \\
\text { Bakri balloon available at } 61 \% \\
\text { of OB/GYNs' institutions }\end{array}$ \\
\hline
\end{tabular}

As $P$ PH presentations progress in severity, treatment strategies begin to diverge and variations in $O B / G Y N s^{\prime}$ approaches to management begin to emerge; clear majority responses are no longer obvious. $\downarrow$

Vaginal delivery; PPH secondary to uterine atony; all uterotonics administered; PPH persists; EBL = 1.5 liters; Patient desires future fertility

Interventional radiology for

embolization

rFVIIa

Too risky

Bakri balloon placement

Vaginal delivery; PPH secondary to uterine atony; massage, compression, and all uterotonics administered; PPH persists;

Consultation with HEM
EBL = 2 liters; hemodynamic instability

$47 \%$

$34 \%$

$17 \%$

$51 \%$

$43 \%$

$4 \%$

Vaginal delivery; PPH secondary to uterine atony; massage, compression, and all uterotonics administered; PPH persists;

EBL = 2 liters; hemodynamic instability;

Determination that blook product replacement is necessary

\section{Empirical Transfusion of Platelets}

Vaginal delivery; PPH secondary to uterine atony; massage, compression, and all uterotonics administered;

EBL $\geq 2$ liters; evidence of significant hemodynamic instability; patient transfused (6 units PRBC, 4 units FFP, 1 unit platelets)

\section{Blood Product Replacement}

Management Strategy:

4 Units PRBC: 1 Unit FFP

Other replacement ratios

Subsequent to platelet count After 4 units FFP

$36 \%$ $29 \%$

Hysterectomy

Other surgical interventions rFVIIa
$42 \%$

$49 \%$

$9 \%$
Q \#27 Question posed with assumption that interventional radiology for embolization and rFVIIa are both available to OB/GYN

$\mathrm{Q}$ \#28

Indication of trigger for hematology consult

$\mathrm{Q} \# 29$

Notable differences in OB/GYNs' responses

Q \#32

Huge variance in OB/GYNs' responses

Q \#33

Suggests that this may be the trigger for potential use of NovoSeven ${ }^{\circledR}$ in refractory PPH if $O B / G Y N$ is familiar with rFVIIa (NOTE: $44 \%$ and $43 \%$ of OB/GYNs are unfamiliar or only slightly familiar with rFVIIa) 
embolization (15\%), B-Lynch compression suture after laparotomy (11\%), laparotomy for hysterectomy (4\%) and administration of rFVIIa (2\%) (Table 4).

For the same scenario with the patient expressing a desire for uterine preservation (Appendix 1, Q \#27), OB/GYNs were more varied in their responses with likely approaches including 47\% IR for embolization, 34\% administration of rFVIIa, and 17\% reporting that alternatives for preserving fertility were too risky and they preferred laparotomy for bilateral hypogastric artery ligation (12\%) or laparotomy to perform hysterectomy (5\%) (Table 4).

As PPH progressed with EBL=2 liters and hemodynamic instability (but no indication that the patient desired future fertility) (Appendix 1, Q \#28), about half (51\%) of OB/GYNs reported Bakri balloon placement as the next treatment step, and $4 \%$ indicated they would consult a hematologist at that point (Table 4).

Once it had been determined that blood replacement was necessary, there was considerable variability in reported blood product replacement management strategies (Table 4) with 57\% preferring a ratio of 4 units PRBC: 1 unit FFP and the remaining 43\% choosing other replacement ratios. There was also large variability in OB/GYNs' responses to empirical transfusion of platelets in this setting: $36 \%$ reported that they transfuse only after obtaining a platelet count and the remainder after 1, 2, 3 or 4 units FFP. Hematologists were also asked this question; $50 \%$ responded that they would transfuse only after obtaining a platelet count.

Once PPH had progressed to EBL > 2 liters, with evidence of significant hemodynamic instability, and following patient transfusion (6 units PRBC, 4 units FFP, 1 unit platelets), OB/GYNs were quite varied in their approach to treatment (Figure 5). Forty-two percent of OB/GYNs reported their next treatment step would be hysterectomy, $49 \%$ were divided between other surgical interventions, and 9\% reported rFVIIa as their next step. In contrast, $40 \%$ of HEMs reported rFVIIa while only $27 \%$ reported hysterectomy as the next treatment step in this scenario (Figure 5).

\subsection{Additional Hypothetical Case Scenarios}

Two additional hypothetical PPH patient scenarios were presented to both OB/GYNs and HEMs with modified treatment choices. OB/GYNs were given choices of consulting HEMs and "other"; HEMs were not given these choices. Obstetrical treatment procedures (B-Lynch, bilateral hypogastric artery ligation, hysterectomy) were combined into one option for HEMs but presented individually to OB/GYNs. Options for both types of providers included administering rFVIIa and performing uterine artery embolization by interventional radiology.

In the first of these case scenarios (appendix 1, Q \#34 \& \#37), the patient was a 31 y/o G2 P1001 at $36+1$ weeks who was presenting for elective repeat Cesarean delivery with a desire for future fertility. (Figure 6) She had placenta previa with suspected placenta accreta. The OB placed balloon catheters to minimize blood loss at

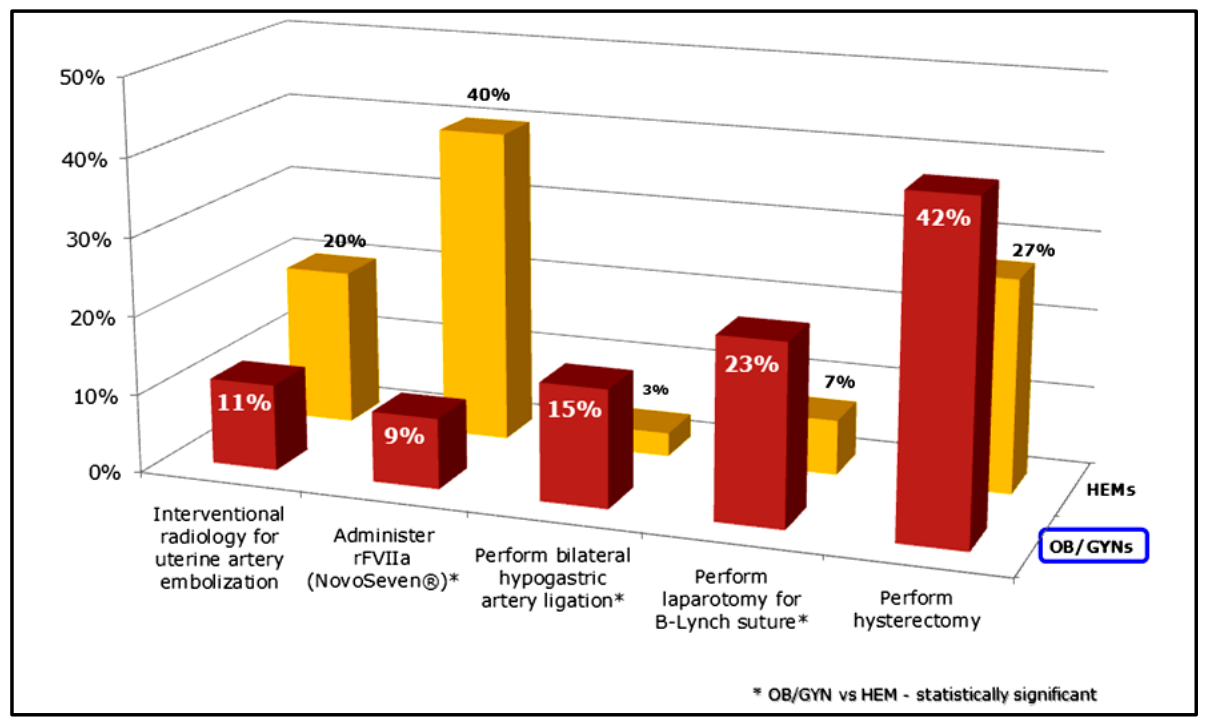

Figure 5. "Next step" intervention in setting of continued hemorrhage subsequent to massive transfusion $(\mathrm{n}=250)$. 


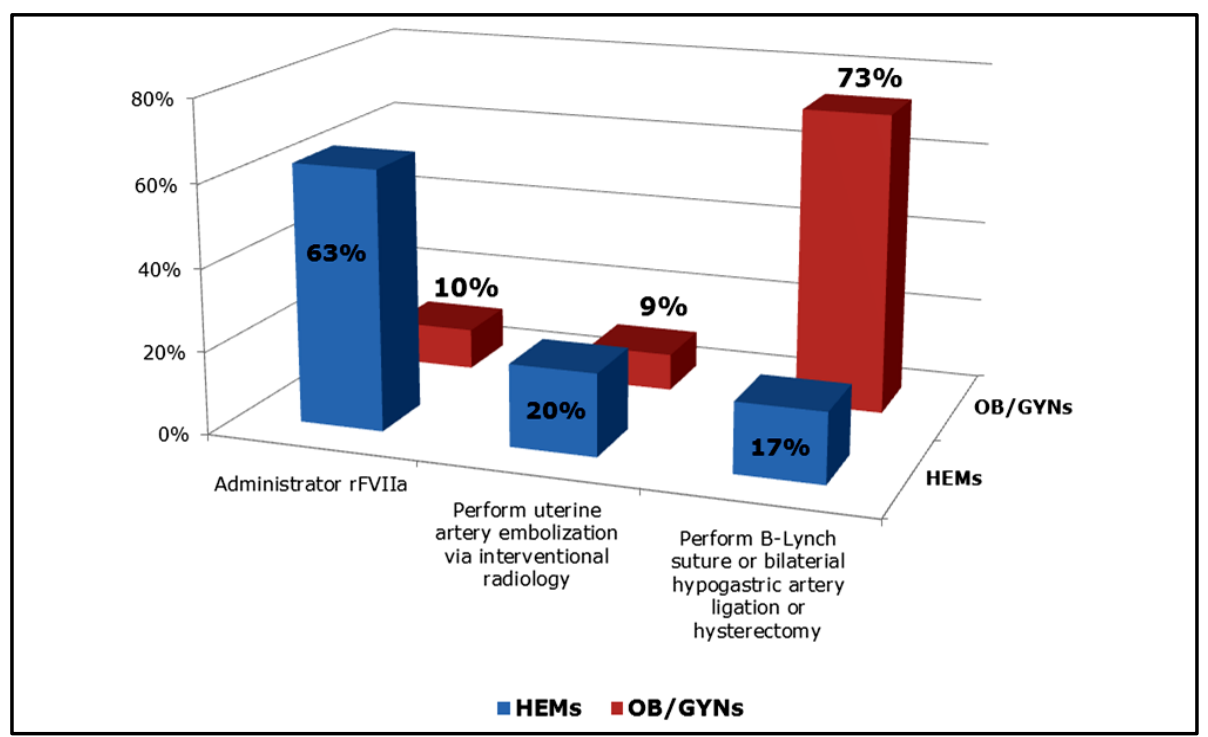

Figure 6. Hypothetical PPH Patient Case \#1, Reported “Next Step” Intervention by OB/GYNs and HEMs $(n=250)$.

delivery. The placenta was removed intact with difficulty after delivery of the fetus with EBL $=4$ liters and evidence of coagulopathy. The patient remained hemodynamically unstable despite blood replacement via a massive transfusion protocol. "Next step" interventions varied dramatically between OB/GYNs and HEMs. Nearly three-quarters (73\%) of OB/GYNs would perform an obstetrical intervention (32\% hysterectomy, 22\% B-Lynch suture, $15 \%$ bilateral artery ligation); only $13 \%$ would choose rFVIIa and 10\% IR embolization. In contrast, nearly two-thirds (63\%) of HEMs would choose rFVIIa, 20\% prefer IR embolization, and $17 \%$ would recommend an obstetrical procedure as the next treatment step (Figure 6).

The next patient scenario (Appendix 1, Q \#35 \& \#36) described a 35 y/o G5 P2113 at 29 weeks presenting with severe preeclampsia and thrombocytopenia, platelet count 105,000, and mildly elevated liver function tests (Figure 7). Antenatal corticosteroids were administered to enhance fetal lung maturation but severe hypertension developed at 23 hours into the hospitalization. The patient was induced and a successful vaginal delivery was achieved. Profound uterine atony required discontinuation of magnesium sulfate given for seizure prophylaxis. Despite uterine massage and appropriate uterotonics, massive PPH with EBL $=3$ liters and hemodynamic instability (partially corrected with blood product replacement) ensued. A majority of OB/GYNs (53\%) would perform an obstetrical procedure as the next treatment step (28\% hysterectomy, $18 \%$ B-Lynch suture, $7 \%$ bilateral artery ligation), $16 \%$ would administer rFVIIa, and $21 \%$ would perform IR embolization. HEMs, in contrast, preferred administering rFVIIa (44\%), followed by IR embolization (33\%) and obstetrical procedures (23\%) (Figure 7).

\subsection{Experience with and Knowledge of rFVIIa}

Several questions on the survey assessed physicians' level of experience with rFVIIa. A large majority of OB/GYNs (87\%) report that they have little or no familiarity with rFVIIa (Table 5). Comparatively, only $20 \%$ of HEMs lack familiarity with rFVIIa (Table 5). In addition, 83\% of OB/GYNs report that they are unfamiliar with the initial dose of rFVIIa (Figure 8). Those with high familiarity with rFVIIa were younger ( $\mu=41.6+5.4$ years) than those with medium $(48.0+8.8)$ or low $(48.2+7.2)$ familiarity $(p<0.05$, data not shown). Notably, $23 \%$ of HEMs and $26 \%$ of OB/GYNs who reported a dose of rFVIIa prefer an initial dose of $40 \mathrm{mcgs} / \mathrm{kg}$. Satisfaction and perceived efficacy of rFVIIa for PPH is highly dependent on familiarity with the treatment (Table 6). A large majority (77\%) of physicians who are most familiar with rRVIIa as treatment for postpartum hemorrhage reported being very or extremely satisfied with the agent for this indication (Table 6). However, a large majority of OB/GYNs (67\%), and notably more than a quarter of HEMs (27\%), are unable to assess rFVIIa's efficacy in the PPH setting due to limited experience or unfamiliarity. Most hematologists (93\%) report being the entity who typically orders rFVIIa in the PPH setting in their hospitals (Figure 9). OB/GYNs expressed much more 


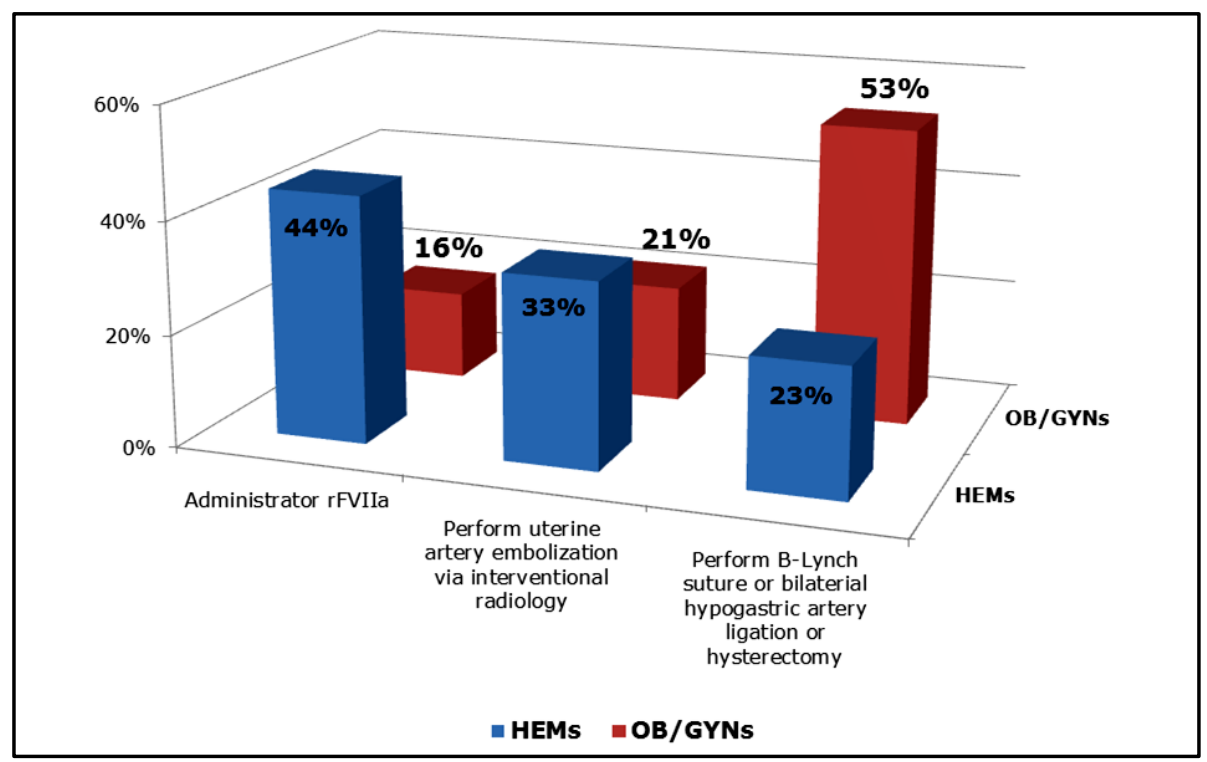

Figure 7. Hypothetical PPH patient case \#2, reported “next step” intervention by OB/GYNs and HEMs.

Table 5. Experience with rFVIIa for treating PPH among OB/GYNs and HEMs.

\begin{tabular}{|c|c|c|c|c|c|c|}
\hline & \multicolumn{2}{|c|}{ Familiarity with rFVIIa } & \multicolumn{2}{|c|}{ Satisfaction with rFVIIa for PPH } & \multicolumn{2}{|c|}{ Efficacy of rFVIIa for treating PPH } \\
\hline & $\begin{array}{l}\text { OB/GYNs } \\
(n=220) \%\end{array}$ & $\begin{array}{c}\text { HEMs } \\
(n=30) \%\end{array}$ & $\begin{array}{c}\text { OB/GYNs } \\
(n=220) \%\end{array}$ & $\begin{array}{c}\text { HEMs } \\
(n=30) \%\end{array}$ & $\begin{array}{l}\text { OB/GYNs } \\
(n=220) \%\end{array}$ & $\begin{array}{c}\text { HEMs } \\
(n=30) \%\end{array}$ \\
\hline Extremely & 0.5 & 10.0 & 0.0 & 3.3 & 0.9 & 6.7 \\
\hline Very & 1.4 & 33.3 & 7.7 & 36.7 & 13.6 & 46.7 \\
\hline Moderately & 11.4 & 36.7 & 6.8 & 33.3 & 14.1 & 20.0 \\
\hline Slightly & 43.2 & 13.3 & 2.7 & 3.3 & 4.1 & 0.0 \\
\hline Not at all & 43.6 & 6.7 & 0.0 & 0.0 & 0.0 & 0.0 \\
\hline Can’t say & $\mathrm{n} / \mathrm{a}$ & $\mathrm{n} / \mathrm{a}$ & 82.7 & 23.3 & 67.3 & 26.7 \\
\hline
\end{tabular}

Note: significant $(p<0.05)$ differences between OB/GYNs and HEMs for each of these three questions.

uncertainty about who typically orders rFVIIA: only $21 \%$ report hematologist, $17 \%$ the managing OB, $26 \%$ didn't know and 23\% reported that the drug was not available at their hospital (Figure 9). Nearly half of the OB/GYNs (45\%) expect that their use of rFVIIa will increase in the next three years. Mean age is significantly lower for OB/GYNs who had high familiarity with rFVIIa (41.6 years) compared to those with medium (48.0) or low (48.2) familiarity (Table 6).

\section{Discussion}

The survey provides a contemporary view of national management trends for postpartum hemorrhage. The survey was restricted to board certified obstetrician-gynecologists and hematologists, which ensured that physicians completing the survey have had significant experience following their residency training. Evidence-based practice guidelines have been published regarding the management of the third stage of labor, [23] and the survey addressed this aspect of delivery management. Despite this, half of OB/GYNs vary in their approach to delivery of the placenta following a routine vaginal delivery.

Not surprisingly, initial management strategies for mild postpartum hemorrhage due to atony were consistent among obstetricians. Initial management consisted of uterine massage, continuation of oxytocin, and administra- 
Table 6. Reported satisfaction with and efficacy of rFVIIa for PPH by familiarity with rFVIIa.

\begin{tabular}{|c|c|c|c|c|c|c|}
\hline & \multicolumn{3}{|c|}{ Satisfaction with rFVIIa for PPH } & \multicolumn{3}{|c|}{ Efficacy of rFVIIa for PPH } \\
\hline & High familiarity & Moderate familiarity & Low familiarity & High familiarity & Moderate familiarity & Low familiarity \\
\hline & $\%$ & $\%$ & $\%$ & $\%$ & $\%$ & $\%$ \\
\hline Extremely & 5.9 & 0 & 0 & 11.8 & 2.8 & 0.5 \\
\hline Very & 70.6 & 27.8 & 3 & 64.7 & 38.9 & 9.6 \\
\hline Moderately & 17.6 & 36.1 & 4.6 & 17.6 & 41.7 & 9.6 \\
\hline Slightly & 0 & 5.6 & 2.5 & 0 & 2.8 & 4.1 \\
\hline Not at all & 0 & 0 & 0 & 0 & 0 & 0 \\
\hline Can’t say & 5.9 & 30.6 & 89.8 & 5.9 & 13.9 & 76.1 \\
\hline
\end{tabular}

Note: significant $(p<0.05)$ differences between OB/GYNs and HEMs for each of these two questions by rFVIIa familiarity.

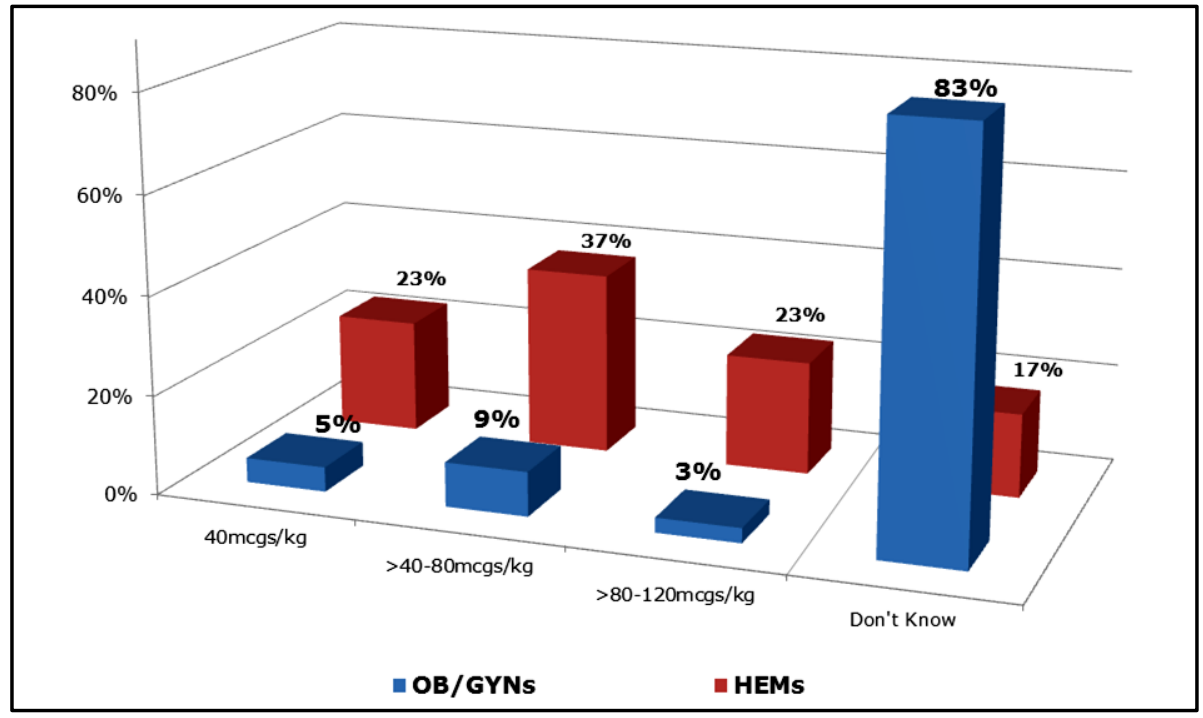

Figure 8. Reported initial dose of rFVIIa in the PPH setting among OB/GYNs and HEMs.

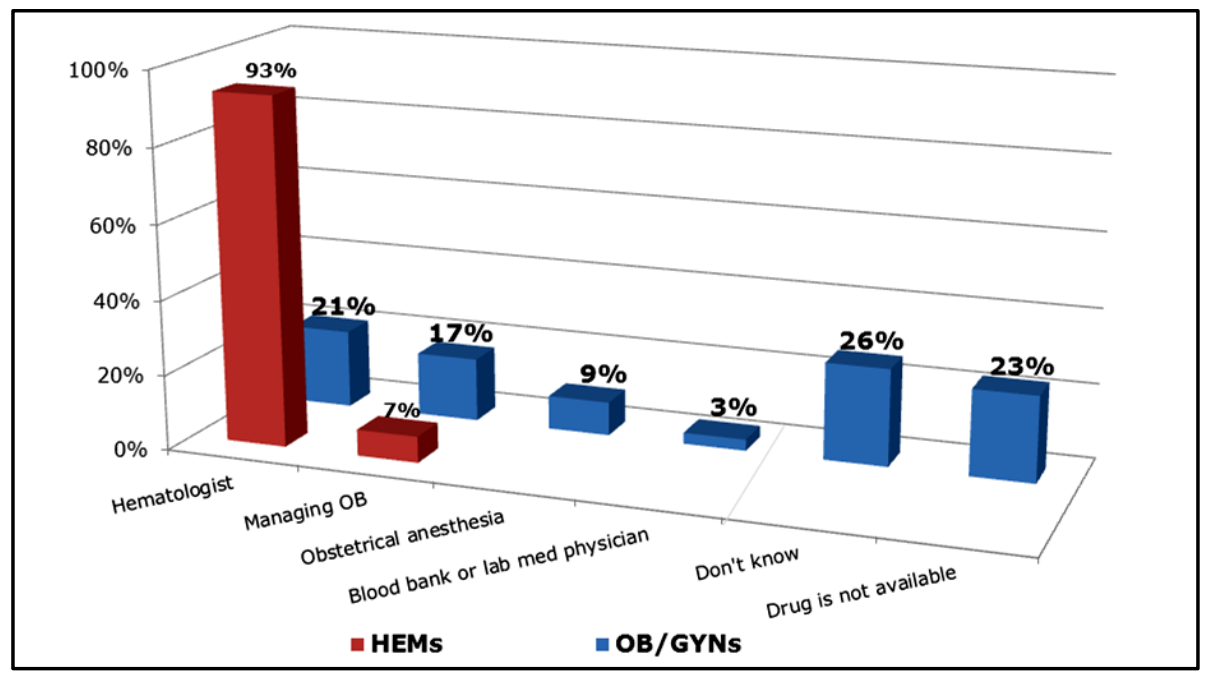

Figure 9. Entity who typically orders rFVIIa in PPH setting among OB/GYNs and HEMs. 
tion IVvolume replacement, followed by methylergonovine. When these measures fail to control hemorrhage and blood loss increases to 1.5 or 2 liters, there is less consistency with management strategies, but about half of OB/GYNs would proceed with the Bakri balloon. B-Lynch compression sutures and IR embolization procedures are the most common next steps. With blood loss of $>2$ liters accompanied by significant hemodynamic instability and after transfusion of blood products, surgical options predominate (hysterectomy, 42\%). Only a small minority of OB/GYNs reported using rFVIIa in the setting (9\%); however, those who do use rFVIIa have a favorable view of it. When fertility preservation is requested by the patient, use of rFVIIa and embolization procedures (assuming available) would reportedly increase.

In the setting of massive postpartum hemorrhage in association with placental abnormalities (previa, accreta), there is even greater variability in terms of management by OB/GYNs after uterotonic agent administration and the institution of a massive transfusion protocol. When the scenario of postpartum hemorrhage involved severe preterm preeclampsia, management with hysterectomy, embolization, and B-Lynch procedures still predominate, but rFVIIa usage increases to $16 \%$. Few OB/GYNs have the full complement of interventions available to them, and this restriction presumably influences local management practices. When HEMs are posed with the same massive PPH scenarios, their next treatment step was predominately administration of rFVIIa. This stark difference in treatment strategy may reflect differences in familiarity with rFVIIa.

A strength of this survey is that we selected a random sample of 1800 physicians from a national database maintained by the American Medical Association to help ensure representativeness. However, a potential limitation of the study is that the results may not be representative of the entire national population of obstetrician gynecologists and hematologists. Despite the apparently low response rate (16\%: 285 of 1800), the survey was designed to be closed after 200 eligible surveys were completed. It is possible, however, that those who completed the surveys were not representative of the 1800 randomly selected. Nonetheless, the survey provides insight into the current treatment practices for postpartum hemorrhage in the US.

A potentially important, relatively new non-invasive treatment option for postpartum hemorrhage is rFVIIa. Our survey found that over three-quarters of physicians who are most familiar with rFVIIa are very satisfied with its effectiveness in the PPH setting, suggesting the potential importance of increasing knowledge of and experience with rFVIIa. However, we also found that a large majority (87\%) of OB/GYNs have little or no familiarity with rFVIIa, compared to only a fifth of HEMs. One obvious barrier to use of rFVIIa is that a quarter of the OB/GYNs did not even know who is responsible for ordering rFVIIa in their hospitals. In addition, the majority of OB/GYNs are unfamiliar with the initial dose of rFVIIa. Notably, nearly a quarter of both HEMs and OB/GYNs who reported a dose of rFVIIa responded that they preferred an initial dose of $40 \mathrm{mcgs} / \mathrm{kg}$, a dose that may be suboptimal in the setting of postpartum hemorrhage (and thus may affect perceptions of efficacy) [13] [14] [18] [24]. Despite these limitations, nearly half of the OB/GYNs expect that their use of rFVIIa to treat PPH will increase in the next three years.

Our survey demonstrates that conventional blood product replacement continues to dominate clinical practice. A ratio of 4 units packed red blood cells to 1 unit of fresh frozen plasma is practiced by the majority of respondents, but the other nearly half of respondents used other ratios. Just over a third of OB/GYN respondents would transfuse platelets only after a platelet count is obtained, compared to $50 \%$ for HEMs. On a related note, less than half of OB/GYNs' postpartum hemorrhage patients receive a hematological evaluation, while a large majority of HEMs' patients receive an evaluation.

The findings from this survey demonstrate the need for better guidelines to manage postpartum hemorrhage, particularly in common clinical scenarios facing obstetrician-gynecologists. Evidence-based practice guidelines regarding management of the third stage of labor should be emphasized. However, despite the significance of postpartum hemorrhage to maternal health, there is a lack of adequate clinical trial data to guide physicians in managing their patients [25]. The first step in addressing postpartum hemorrhage is to accurately estimate the severity. Estimates of blood loss in the clinical setting generally lack precision. Fuller has provided a helpful classification [26]. There are essentially three categories of postpartum hemorrhage (mild, moderate and severe). Acceptable blood loss would be described as no postpartum hemorrhage (American College of Surgeons (ACS) class I; asymptomatic; Estimated Blood Loss (EBL) 750 ml, \% EBL 10\% - 15\%; no treatment is needed). Mild postpartum hemorrhage is described as: ACS class II; signs \& symptoms include tachycardia and hypotension; EBL 750 - $1500 \mathrm{ml}$, \% EBL 15\% - 25\%; treatment consists of volume replacement. Moderate postpartum hemorrhage is described as: ACS class III; signs \& symptoms include Pulse 100 - 120, SBP 80 - 100, oliguria; EBL 1500 - $2000 \mathrm{ml}$, \% EBL 25\% - 40\%; treatment typically involves transfusion of blood products. Severe 
postpartum hemorrhage is described as: ACS class IV; signs \& symptoms include Pulse 120 - 140, SBP < 80, anuria; EBL > $2000 \mathrm{ml}$, \% EBL > 40\%; treatment involves transfusion and activation of a massive transfusion protocol.

Less than half of participating OB/GYNs are affiliated with hospitals that have a massive transfusion protocol to treat severe PPH. Treatment guidelines, therefore, are likely to be largely unstructured and individualized. This may account for the large practice variations by respondents of this survey. Because the majority of women who develop postpartum hemorrhage have no identified risk factors, active management of the third stage of labor has been advocated to ameliorate preventable hemorrhage. Expectant management of the third stage has been associated with a three times higher rate of postpartum hemorrhage when compared to active management [27]. Currently, there is a preponderance of evidence demonstrating that the active management of the third stage of labor (uterine massage, controlled traction of the umbilical cord and use of medication to effect uterine contractions) is effective in preventing the need for a higher level of care in actively managed patients [23]. However, postpartum hemorrhage is still a common scenario facing obstetrical providers which will require active management of the patient to stem the hemorrhage. The obstetrical provider must rapidly consider the possible etiologies for postpartum hemorrhage, while also considering possible diagnostic tests and treatment, all of which must occur simultaneously.

\section{Conclusion}

A well-considered transfusion protocol, created by a multidisciplinary group consisting of obstetrician gynecologists, obstetrical anesthesiologists, hematologists, and blood bank/laboratory medicine specialists, will offer local options. A massive transfusion protocol should be available in each hospital and known to all relevant care providers. The creation of such a protocol is beyond the scope of this manuscript. It is important to remember that undiagnosed bleeding disorders can be responsible for postpartum hemorrhage, and von Willebrand disease is the most common bleeding entity discovered in this setting. A summary of available approaches to treat postpartum hemorrhage has been published [28], but present guidelines can be improved by providing more specific algorithms, including the array of presently available interventions. Thromboelastography provides point of care monitoring to assess hemostasis in postpartum hemorrhage [29]. Promising interventions include tranexamic acid and fibrinogen concentrate and both are being prospectively evaluated in clinical trials [30] [31].

\section{Acknowledgements}

The authors acknowledge Debra J. Boyd, PhD, PsymedInc (Valley Forge, PA) for assistance in the design, administration, and analysis of the survey. This study was funded by an investigator (MJP)-initiated research grant from Novo Nordisk ${ }^{\circledR}$ (Bagsvaerd, Denmark). Competing interests: MJP received an honorarium for participating on an International Round Table on postpartum hemorrhage, sponsored by CSL Behring (Marburg, Germany). The other authors (EWT and MJW) declare they have no competing interests. The views expressed herein are those of the author(s) and do not reflect the official policy of the Department of the Army, Department of Defense, or the U.S. Government.

\section{References}

[1] Cohen, W.R. (2006) Hemorrhagic Shock in Obstetrics. Journal of Perinatal Medicine, 34, 263-271. http://dx.doi.org/10.1515/JPM.2006.051

[2] Johns, B., Sigurbjornsdottir, K., Fogstad, H., Zupan, J., Mathai, M. and Tan-Torres Edejer, T. (2007) Estimated Global Resources Needed to Attain Universal Coverage of Maternal and Newborn Health Services. Bull World Health Organ, 85, 256-263. http://dx.doi.org/10.2471/BLT.06.032037

[3] Mathai, M., Gulmezoglu, A.M. and Hill, S. (2007) Saving Women’s Lives: Evidence-Based Recommendations for the Prevention of Postpartum Haemorrhage. Bull World Health Organ, 85, 322-323.

[4] Health Advisory (2004) Prevention of Maternal Deaths through Improved Management of Hemorrhage www.health.state.ny.us/professionals/protocols_and_guidelines/maternal_hemorrhage/

[5] American Academy of Pediatrics and American College of Obstetricians and Gynecologists (2007) Guidelines for Perinatal Care. 6th Edition, American Academy of Pediatrics and the American College of Obstetricians and Gynecologists; Washington DC.

[6] Bouwmeester, F.W., Bolte, A.C. and van Geijn, H.P. (2005) Pharmacological and Surgical Therapy for Primary Post- 
partum Hemorrhage. Current Pharmaceutical Design, 11, 759-773. http://dx.doi.org/10.2174/1381612053381882

[7] Haljamae, H. (1993) The Pathophysiology of Shock. Acta Anaesthesiologica Scandinavica, 98, 3-6. http://dx.doi.org/10.1111/j.1399-6576.1991.tb05064.x

[8] Velanovich, V. (1989) Crystalloid versus Colloid Fluid Resuscitation: A Meta-Analysis of Mortality. Surgery, 105, 65-71.

[9] Schwartz, S.I., Shires, G.T. and Spencer, F.C. (1999) Hemostasis, Surgical Bleeding, and Transfusion. In: Anonymous, Ed., Schwartz's Principles of Surgery, 7th Edition, McGraw-Hill Professional, New York, 107-108.

[10] Matot, I., Einav, S., Goodman, S., Zeldin, A., Weissman, C. and Elchalal, U. (2004) A Survey of Physicians’ Attitudes toward Blood Transfusion in Patients Undergoing Cesarean Section. American Journal of Obstetic Gynecology, 190, 462-467. http://dx.doi.org/10.1016/j.ajog.2003.07.028

[11] Santoso, J.T., Saunders, B.A. and Grosshart, K. (2005) Massive Blood Loss and Transfusion in Obstetrics and Gynecology. Obstetrical \& Gynecological Survey, 60, 827-837. http://dx.doi.org/10.1097/01.ogx.0000189154.98227.4b

[12] Burtelow, M., Riley, E., Druzin, M., Fontaine, M., Viele, M. and Goodnough, L.T. (2007) How We Treat: Management of Life-Threatening Primary Postpartum Hemorrhage with a Standardized Massive Transfusion Protocol. Transfusion, 47, 1564-1572. http://dx.doi.org/10.1111/j.1537-2995.2007.01404.x

[13] Bouma, L.S., Bolte, A.C. and van Geijn, H.P. (2008) Use of Recombinant Activated Factor VII in Massive Postpartum Haemorrhage. The European Journal of Obstetrics \& Gynecology and Reproductive Biology, 137, 172-177. http://dx.doi.org/10.1016/j.ejogrb.2007.06.022

[14] Bouwmeester, F.W., Jonkhoff, A.R., Verheijen, R.H. and van Geijn, H.P. (2003) Successful Treatment of Life-Threatening Postpartum Hemorrhage with Recombinant Activated Factor VII. Obstetrics \& Gynecology, 101, 1174-1176. http://dx.doi.org/10.1016/S0029-7844(03)00350-8

[15] Brice, A., Hilbert, U., Roger-Christoph, S., Fernandez, H., Dumenil, A.S., Descorps-Declere, A., Mercier, F. and Benhamou, D. (2004) Recombinant Activated Factor VII as a Life-Saving Therapy for Severe Postpartum Haemorrhage Unresponsive to Conservative Traditional Management. Annales Françaises d'Anesthésie et de Réanimation, 23, 10841088. http://dx.doi.org/10.1016/j.annfar.2004.09.005

[16] Haynes, J., Laffan, M. and Plaat, F. (2007) Use of Recombinant Activated Factor VII in Massive Obstetric Haemorrhage. International Journal of Obstetric Anesthesia, 16, 40-49. http://dx.doi.org/10.1016/j.ijoa.2006.07.005

[17] Sobieszczyk, S., Breborowicz, G.H., Platicanov, V., Tanchev, S. and Kessler, C.M. (2006) Recombinant Factor VIIa in the Management of Postpartum Bleeds: An Audit of Clinical Use. Acta Obstetricia et Gynecologica Scandinavica, 85, 1239-1247. http://dx.doi.org/10.1080/00016340600855839

[18] Hossain, N., Shamsi, T., Haider, S., Soomro, N., Khan, N.H., Memon, G.U., Farzana, T., Ansari, S., Triche, E.W., Kuczynski, E., et al. (2007) Use of Recombinant Activated Factor VII for Massive Postpartum Hemorrhage. Acta Obstetricia et Gynecologica Scandinavica, 86, 1200-1206. http://dx.doi.org/10.1080/00016340701619324

[19] Phillips, L.E., McLintock, C., Pollock, W., Gatt, S., Popham, P., Jankelowitz, G., Ogle, R. and Cameron, P.A. (2009) Australian and New Zealand Haemostasis Registry: Recombinant Activated Factor VII in Obstetric Hemorrhage: Experiences from the Australian and New Zealand Haemostasis Registry. Anesthesia \& Analgesia, 109, 1908-1915. http://dx.doi.org/10.1213/ANE.0b013e3181c039e6

[20] Perkins, J.G., Cap, A.P., Weiss, B.M., Reid, T.J. and Bolan, C.D. (2008) Massive Transfusion and Nonsurgical Hemostatic Agents. Critical Care Medicine, 36, S325-S339. http://dx.doi.org/10.1097/CCM.0b013e31817e2ec5

[21] Malone, D.L., Hess, J.R. and Fingerhut, A. (2006) Massive Transfusion Practices around the Globe and a Suggestion for a Common Massive Transfusion Protocol. Journal of Trauma, 60, S91-S96. http://dx.doi.org/10.1097/01.ta.0000199549.80731.e6

[22] Cameron, C.A., Roberts, C.L., Bell, J. and Fischer, W. (2007) Getting an Evidence-Based Post-Partum Haemorrhage Policy into Practice. Australian and New Zealand Journal of Obstetrics and Gynaecology, 47, 169-175. http://dx.doi.org/10.1111/j.1479-828X.2007.00713.x

[23] Rajan, P.V. and Wing, D.A. (2010) Postpartum Hemorrhage: Evidence-Based Medical Interventions for Prevention and Treatment. Clinical Obstetrics \& Gynecology, 53, 165-181. http://dx.doi.org/10.1097/GRF.0b013e3181ce0965

[24] Heilmann, L., Wild, C., Hojnacki, B. and Pollow, K. (2006) Successful Treatment of Life-Threatening Bleeding after Cesarean Section with Recombinant Activated Factor VII. Clinical and Applied Thrombosis/Hemostasis, 12, $227-229$. http://dx.doi.org/10.1177/107602960601200213

[25] Levy, J.H., Dutton, R.P., Hemphill III, J.C., Shander, A., Cooper, D., Paidas, M.J., Kessler, C.M., Holcomb, J.B. and Lawson, J.H. (2010) Hemostasis Summit Participants: Multidisciplinary Approach to the Challenge of Hemostasis. Anesthesia \& Analgesia, 110, 354-364. http://dx.doi.org/10.1213/ANE.0b013e3181c84ba5

[26] Fuller, A.J. and Bucklin, B. (2007) Blood Component Therapy in Obstetrics. Obstetrics and Gynecology Clinics of North 
America, 34, 443-458. http://dx.doi.org/10.1016/j.ogc.2007.06.003

[27] Prendiville, W.J., Harding, J.E., Elbourne, D.R. and Stirrat, G.M. (1988) The Bristol Third Stage Trial: Active versus Physiological Management of Third Stage of Labour. British Medical Journal, 297, 1295-1300. http://dx.doi.org/10.1136/bmj.297.6659.1295

[28] American College of Obstetricians and Gynecologists (2006) ACOG Practice Bulletin: Clinical Management Guidelines for Obstetrician-Gynecologists, Number 76, October 2006: Postpartum Hemorrhage. Obstetrics and Gynecology, 108, 1039-1047.

[29] Pacheco, L.D., Saade, G.R., Gei, A.F. and Hankins, G.D. (2011) Cutting-Edge Advances in the Medical Management of Obstetrical Hemorrhage. American Journal of Obstetrics \& Gynecology, 205, 526-532. http://dx.doi.org/10.1016/j.ajog.2011.06.009

[30] Shakur, H., Elbourne, D., Gülmezoglu, M., Alfirevic, Z., Ronsmans, C., Allen, E. and Roberts, I. (2010) The WOMAN Trial (World Maternal Antifibrinolytic Trial): Tranexamic Acid for the Treatment of Postpartum Haemorrhage: An International Randomised, Double Blind Placebo Controlled Trial. Trials, 11, 40. http://dx.doi.org/10.1186/1745-6215-11-40

[31] Wikkelsoe, A.J., Afshari, A., Stensballe, J., Langhoff-Roos, J., Albrechtsen, C., Ekelund, K., Hanke, G., Sharif, H.F., Mitchell, A.U., Svare, J., et al. (2012) The FIB-PPH Trial: Fibrinogen Concentrate as Initial Treatment for Postpartum Haemorrhage: Study Protocol for a Randomised Controlled Trial. Trials, 13, 110. http://dx.doi.org/10.1186/1745-6215-13-110 